Bundesgesundheitsbl 2019 $\cdot 62: 533$

https://doi.org/10.1007/s00103-019-02914-0

c) Springer-Verlag GmbH Deutschland, ein Teil von Springer Nature 2019

Bekanntmachung des Umweltbundesamtes

\title{
Neuberufung der Mitglieder der Schwimm- und Badebeckenwasserkommission beim Umweltbundesamt
}

Auf der Grundlage des $\$ 40$ Infektionsschutzgesetz berief das Bundesministerium für Gesundheit am 8. Januar 2019 folgende Experten zu Mitgliedern der Schwimm- und Badebeckenwasserkommission beim Umweltbundesamt für die Berufungsperiode 2019-2023:

- Herrn Dr. Dirk P. Dygutsch, Dr. Nüsken Chemie GmbH, Kamen

- Herrn Priv.-Doz. Dr. Lothar Erdinger, Universitätsklinikum, Heidelberg

- Frau Prof. Dr. Christiane Höller, Bayerisches Landesamt für Gesundheit und Lebensmittelsicherheit, Oberschleißheim

- Herrn Albert Karras, Gesundheitsamt Rhein-Neckar-Kreis, Heidelberg

- Frau Dr. Katrin Luden, Niedersächsisches Landesgesundheitsamt, Aurich

- Herrn Dr. Axel Matthiessen, Universitätsklinikum Schleswig-Holstein, Kiel

- Frau Gudrun Petzold, Ministerium für Soziales, Gesundheit, Jugend, Familie und Senioren des Landes Schleswig-Holstein, Kiel

- Frau Dr. Nicole Riedle, Balneatechnik $\mathrm{GmbH}$, Wiesbaden

- Herrn Jörg Rosbach, Bäder Betriebe Frankfurt GmbH, Frankfurt am Main

- Herrn Heiko Schulze, Kommunale Wasserwerke Leipzig GmbH, Leipzig

- Herrn Michael Stumpf, Bundesverband der Hygieneinspektoren, Saarlouis

- Herrn Priv.-Doz. Dr. Georg-Joachim Tuschewitzki, Hygieneinstitut des Ruhrgebietes, Gelsenkirchen

- Herrn Tim Westphal, Gesundheitsamt Frankfurt am Main
Die Kommissionsmitglieder wählten Frau Prof. Dr. Christiane Höller zur Vorsitzenden und Herrn Priv.-Doz. Dr. Lothar Erdinger zum stellvertretenden Vorsitzenden der Schwimm- und Badebeckenwasserkommission.

Der wissenschaftlichen Geschäftsstelle beim Umweltbundesamt wurde die Organisation der Kommissionsarbeit übertragen.

Laut Geschäftsordnung können an den Sitzungen der Schwimm- und Badebeckenwasserkommission (als nicht stimmberechtigt) Vertreterinnen und Vertreter des Bundesministeriums für Gesundheit, des Bundesministeriums für Umwelt, $\mathrm{Na}$ turschutz und Reaktorsicherheit, des Bundesministeriums für Verteidigung, des Umweltbundesamtes sowie der zuständigen Landesbehörden beratend teilnehmen.

(Siehe auch unter: http://www.umweltbundesamt.de/wasser/themen/badebeckenwasser/subbwasserkommission.htm) 